\title{
Subjective memory complaints among patients on sick leave are associated with symptoms of fatigue and anxiety
}

\author{
Julie K. Aasvik ${ }^{*}$, Astrid Woodhouse ${ }^{2,3,4}$, Henrik B. Jacobsen ${ }^{1,2,3}$, \\ Petter C. Borchgrevink ${ }^{1,2,3}$, Tore C. Stiles ${ }^{2,3,5}$ and Nils I. Landro ${ }^{1,3,6}$ \\ ${ }^{1}$ Department of Circulation and Medical Imaging, Faculty of Medicine, Norwegian University of Science and Technology, \\ Trondheim, Norway, ${ }^{2}$ Hysnes Rehabilitation Center, St. Olav's University Hospital, Trondheim, Norway, ${ }^{3}$ National \\ Competence Centre for Complex Disorders, St. Olav's University Hospital, Trondheim, Norway, ${ }^{4}$ Department of Public \\ Health and General Practice, Norwegian University of Science of Technology, Trondheim, Norway, ${ }^{5}$ Department of \\ Psychology, Norwegian University of Science and Technology, Trondheim, Norway, ${ }^{6}$ Clinical Neuroscience Group, \\ Department of Psychology, University of Oslo, Oslo, Norway
}

Lorys Castelli,

University of Turin, Italy

Reviewed by:

Paula Goolkasian,

University of North Carolina

at Charlotte, USA

Remko Soer,

University Medical Center

Groningen/Saxion University of Applied Sciences, Netherlands

*Correspondence: Julie K. Aasvik,

Department of Circulation and Medical Imaging, Faculty of Medicine, Norwegian University

of Science and Technology, P.O. Box 8905 MTFS,

7491 Trondheim, Norway julie.k.aasvik@ntnu.no

Specialty section:

This article was submitted to Psychology for Clinical Settings, a section of the journal

Frontiers in Psychology

Received: 07 April 2015

Accepted: 20 August 2015 Published: 08 September 2015

Citation:

Aasvik JK, Woodhouse A, Jacobsen HB, Borchgrevink PC, Stiles TC and Landro NI (2015) Subjective memory complaints among patients on sick leave are associated with symptoms

of fatigue and anxiety.

Front. Psychol. 6:1338. doi: 10.3389/fpsyg.2015.01338
Objective: The aim of this study was to identify symptoms associated with subjective memory complaints (SMCs) among subjects who are currently on sick leave due to symptoms of chronic pain, fatigue, depression, anxiety, and insomnia.

Methods: This was a cross-sectional study, subjects $(n=167)$ who were currently on sick leave were asked to complete an extensive survey consisting of the following: items addressing their sociodemographics, one item from the SF-8 health survey measuring pain, Chalder Fatigue Questionnaire, Hospital Anxiety and Depression Scale, Insomnia Severity Index, and Everyday Memory Questionnaire - Revised. General linear modeling was used to analyze variables associated with SMCs.

Results: Symptoms of fatigue $(p$-value $<0.001)$ and anxiety ( $p$-value $=0.001)$ were uniquely and significantly associated with perceived memory failures. The associations with symptoms of pain, depression, and insomnia were not statistically significant.

Conclusions: Subjective memory complaints should be recognized as part of the complex symptomatology among patients who report multiple symptoms, especially in cases of fatigue and anxiety. Self-report questionnaires measuring perceived memory failures may be a quick and easy way to incorporate and extend this knowledge into clinical practice.

Keywords: cognitive, complaints, concurrent, symptoms, sick leave

\section{Introduction}

Subjective memory complaints (SMCs) have been associated with conditions of chronic pain (McCracken and Iverson, 2001; Katz et al., 2004; Munoz and Esteve, 2005; Tesio et al., 2014), fatigue (Cope et al., 1995; Suhr, 2003; Roth et al., 2005), depression (Comijs et al., 2002; Roth et al., 2005; Mowla et al., 2007), and anxiety (O'Boyle et al., 1990; Derouesne et al., 1999) as well as insomnia (Kronholm et al., 2009; Magali et al., 2012). The fact that all of the aforementioned conditions also often co-occur (Smith, 1992; Schotte et al., 2006; Stewart et al., 2006; 
Friedberg, 2010; Lumley et al., 2011) adds to the complexity of studying the nature of SMCs. SMCs include forgetfulness, increased distractibility and reduced mental alertness. Selfperceived memory problems are widespread in the general population, and estimates show that they are prevalent in all age groups and in both genders (Cooper et al., 2011; Holmen et al., 2013). The estimates increase when SMCs are associated with illness (Cockshell and Mathias, 2014).

Subjective memory complaints are considered as one of the major diagnostic criteria's for depression (DSM-5). It has been associated with mild cognitive impairment (Erk et al., 2011) and has been highlighted as a potential marker for future dementia and Alzheimer's disease (Stewart et al., 2011; Stewart, 2012). SMCs have also been associated with work-related exhaustion (Ekstedt and Fagerberg, 2005) and impaired ability to cope with work-related stress (Broadbent et al., 1982). Despite the frequency of SMCs and their potential negative impacts, SMCs remain one of the least recognized and assessed aspects within the field of occupational health psychology.

A number of studies have reported associations between SMCs and depression (McCracken and Iverson, 2001; Comijs et al., 2002; Roth et al., 2005; Mowla et al., 2007). It has been suggested that this relationship might reflect an extension of the emotional distress, low self-esteem, and lack of confidence associated with depression. Such factors might cause tendencies to underestimate one's own performance and, consequently over-report memory failures. Supporting this view, Antikainen et al. (2001) found that mood improvement was associated with reduced SMCs in depressed individuals. Landrø et al. (2013) found higher rates of SMCs in patients with chronic pain, even after adjusting for symptoms of depression. This indicates that the interpretation of SMCs as solely an expression of depressive symptomatology might be somewhat incomplete. In addition, one study (Derouesne et al., 1999) found that the best predictor of the severity of SMCs included symptoms of anxiety. However, although a number of studies have included measures of depression, few have considered co-morbid anxiety.

Studies of SMCs in patients with chronic pain have reported inconsistent results for the associations with pain intensity (Comijs et al., 2002; Munoz and Esteve, 2005; Tesio et al., 2014). Moreover, some studies have found a correspondence between SMCs and pain-related anxiety and pain catastrophizing (McCracken and Iverson, 2001; Munoz and Esteve, 2005), suggesting that it is not just pain intensity per se that is associated with SMCs. Studies examining the associations between chronic pain and SMCs also differ in regards to whether they have included measures of fatigue even though these symptoms often co-occur. Studies on the association between SMCs and insomnia have yielded somewhat contradicting results (Kronholm et al., 2009; Magali et al., 2012).

In general, the multi-factorial nature of SMCs is challenging, and what seems to be lacking is a more comprehensive examination of all relevant symptom dimensions that are associated with SMCs. Identifying key symptoms associated with SMCs might have clinical implications by aiding therapeutic and rehabilitation programs to develop interventions that target the specific conditions that are associated with such complaints. In this study, the symptoms of pain, fatigue, depression, anxiety, and insomnia as well as self-reported memory complaints were assessed in a sample of patients on sick leave who were enrolled in a vocational rehabilitation program. The main aim of the study was to identify which of the five symptoms is significantly associated with subjective memory complaint.

\section{Materials and Methods}

\section{Ethics Statement}

All subjects received information about the study and gave written informed consent before inclusion. The study was approved by Regional Committees for Medical and Health Research Ethics - REC Central, and it was conducted in accordance with the declaration of Helsinki.

\section{Study Design and Setting}

This was a cross-sectional study, conducted during the period of January 2012 until March 2013. Participants were consecutively recruited from an inpatient vocational rehabilitation center in Norway.

General practitioners referred patients who were on sick leave to a 3.5-weeks intervention at a vocational rehabilitation center. All patients were asked to answer a web-based survey before meeting with a multidisciplinary team (physician, psychologist, and physiotherapist) at a designated outpatient clinic to determine if they met the inclusion criteria. The survey included measures of socio-demographics, pain, fatigue, depression, anxiety, sleep disturbances and SMCs. The collected data were used as a source tool by the outpatient and inpatient clinics and were simultaneously included in a research database.

\section{Study Participants}

All participants were on sick leave ( $<8$ weeks) due to chronic pain, fatigue and/or common mental disorders. All participants were between 18-59 years of age. The exclusion criteria were patients with severe mental disorders, (acute psychosis, ongoing manic episode, or suicidal ideation) ongoing substance abuse or addiction, cases of pregnancy, or individuals who were unable to communicate in Norwegian. Considering that this was a returnto-work rehabilitation evaluation, patients were excluded if they did not define returning to work as a personal goal. For further details on the intervention, see (Fimland et al., 2014).

\section{Assessments}

\section{Memory Complaints}

Subjective cognitive complaints are based upon a self-report questionnaire, The Everyday Memory Questionnaire-Revised (EMQ-R; Royle and Lincoln, 2008). The EMQ was originally developed by Sunderland et al. (1983) and later revised (shortened) by Royle and Lincoln (2008). The EMQ-R consists of 13 items, it makes it possible to distinguish between three factors, two of them have been identified as (1) retrieval and (2) attentional tracking, the third has not been identified or labeled. Each question is rated on a 5-point scale ranging from $\mathrm{A}$, scored 
as zero - "Once or less in the last month," to E, scored as four "Once or more in a day." The items were summed, giving a scale from 0 to 52 . Because we chose to focus on general memory functioning we used the total sum score divided by the number of items, following the scoring procedure. Reliability tests on the EMQ have shown a strong internal reliability, with a Cronbach's alpha score of 0.89 (Royle and Lincoln, 2008).

\section{Chronic Pain}

We used one item from the SF- 8 health survey for measuring the intensity of pain (Ware et al., 2001). The subjects were asked "How much bodily pain have you experienced during the last week?" (none, very mild, mild, moderate, severe, or very severe). As this is a single item measure, alpha values are not applicable. The item has shown an intra-class correlation coefficient of 0.66 (95\% CI 0.65-0.67; Landmark et al., 2012).

\section{Depression and Anxiety}

The Hospital Anxiety and Depression Scale (HADS) was used (Zigmond and Snaith, 1983). The fourteen item scale is divided into two sub-scales; one sub-scale scores depression and the other scores anxiety. Each item ranges from 0 to 3 , and the items are summed, giving a score ranging from 0 to 21 in each sub-scale. The psychometric properties of the scale as a self-rating instrument have been validated (Olssøn et al., 2005). In a review of HADS the correlations between the two subscales varied from 0.40 to 0.74 (mean 0.56). Cronbach's alpha for HADS-A varied from 0.68 to 0.93 (mean 0.83) and for HADS-D from 0.67 to 0.90 (mean 0.82; Bjelland et al., 2001).

\section{Fatigue}

Fatigue was measured with The Chalder Fatigue Questionnaire (CFQ Chalder et al., 1993), which consists of 11 questions, reflecting physical, and mental fatigue. Each item has four response categories that are scored bimodally 0-0-1-1. Responses are summed in a scale from 0 to 11 . The scale is both reliable and valid and has been validated for a Norwegian population. Cronbach's alpha has been calculated for all items (range 0.88-0.90). Split half reliability has also been calculated (0.8613 and 0.8466 , respectively) in a previous study (Chalder et al., 1993; Loge et al., 1998; Moriss et al., 1998).

\section{Insomnia}

The Insomnia Severity Index (ISI) was used (Bastien et al., 2001). This is a self-rated questionnaire consisting of seven items designed to assess insomnia severity by the following: difficulty falling asleep, night time awakenings, early morning awakenings, impairment of daytime functioning due to sleep disturbances, noticeability of problems, distress, or worry caused by sleep disturbances, and dissatisfaction with sleep. Each item is rated according to a 5-point scale, ranging from 0 (not at all) to 4 (very much). The items were summed, giving a scale of $0-28$. The ISI is a reliable, valid and sensitive self-report questionnaire, and has been recommended as a screening device and an outcome measure in insomnia treatment and insomnia research. The internal consistency of the ISI was reported to be 0.74 (Bastien et al., 2001; Morin et al., 2011).

\section{Statistical Analyses}

Data was analyzed using the Statistical Package for the Social Sciences (SPSS version 20.0; IBM Corporation, Armonk, NY, USA). To test the association between symptom measures we used Spearman's correlation. General linear modeling (GLM) was used to analyze variables that were associated with SMCs. However, the variables had unacceptable residual plots, and as a result multiple robust regression with Huber's correction was chosen in both unadjusted and adjusted models. Each independent variable (fatigue, pain, anxiety, depression, and insomnia) was first entered in a separate analysis while controlling for age and gender. In post hoc analyses the variables that were significantly associated with SMCs were simultaneously entered while controlling for age and gender in order to assess the unique associations with SMCs.

\section{Results}

\section{Participant Characteristics}

The mean age for the entire group was 41 years ( $S D$ 9.9), and $78.3 \%$ of the subjects were women. Of the 198 patients who were originally included, seven subjects declined to participate, and 8 subjects canceled their rehabilitation intervention and withdrew from the study due to illness or injury. Sixteen subjects had missing data on the Everyday Memory Questionnaire; they were excluded case by case from the analyses. Analyses confirmed that the excluded subjects were similar to the sample population in terms of their symptoms of chronic pain, fatigue, anxiety, depression, and insomnia. The final study population consisted of 167 subjects. The distribution of diagnoses in the sample was: chronic pain $36.8 \%(n=63)$, fatigue $28.7 \%(n=49)$, depression $11.1 \%(n=19)$, anxiety $4.7 \%(n=8)$, both depression and anxiety $4.7 \%(n=8)$, other diagnoses (including obesity, diabetes, hypothyroidism, asthma etc.) $15.2 \%(n=26)$. None of the subjects had a diagnosis of insomnia or sleep-disturbances. $25.7 \%$ ( $n=44)$ were referred based on a description of their health complaints rather than a specific diagnosis. $23.4 \%(n=40)$ of the sample had two diagnoses, $4.7 \%(n=8)$ had three diagnoses,

TABLE 1 | Means and SD of the various symptom measures.

\begin{tabular}{llll}
\hline Self-Report Questionnaires & N & M & SD \\
\hline Everyday Memory Complaints (EMQ-R) & 167 & 1.3 & 0.9 \\
Chronic pain (SF8) & 167 & 3.6 & 1.2 \\
Chalder Fatigue Scale (CFS) & 164 & 8.1 & 2.7 \\
The Hospital Anxiety and Depression Scale (HADS) & & & \\
HADS Anxiety & 167 & 7.5 & 4.1 \\
HADS Depression & 167 & 5.6 & 3.8 \\
Insomnia Severity Index (ISI) & 167 & 10.6 & 5.9
\end{tabular}


underlining the fact that this group of subjects often present multiple, non-specific and complex health-related complaints.

Self-reported complaints regarding everyday memory failures, chronic pain, fatigue, depression, anxiety, and insomnia are described in Table 1. The average score on EMQ-R indicate that our subjects experience multiple kinds of memory failures several times a month, but not on a weekly basis according to the prevailing interpretation of the questionnaire (Royle and Lincoln, 2008). The mean score on the item assessing pain intensity (SF8) indicate (according to the scale) that subjects have experienced mild to moderate pain in the last 4 weeks. The average score on CFQ is above the cut-off at $\geq 5$ used to indicate clinical fatigue (Loge et al., 1998). Subjects score slightly higher on measures of anxiety than depression. Average scores are just below the cut-off at $\geq 8$ used to indicate clinical anxiety and depression (Haug et al., 2004 ). Finally, the average score on ISI is within the range (814) of what constitutes as subthreshold insomnia (Bastien et al., 2001).

Because of the comorbidity when it comes to symptoms of chronic pain, fatigue, depression, anxiety, and insomnia we calculated the inter-correlations between the symptom measures, the results are described in Table 2 . We found a significant correlation between fatigue, depression, anxiety, and insomnia, while pain intensity was significantly correlated with insomnia.

As shown in Table 3, the initial unadjusted analyses showed that both levels of fatigue, anxiety, and depression were significantly associated with SMCs. When these three variables were entered simultaneously with age and gender as covariates in post hoc analyses the levels of fatigue and anxiety remained significantly associated with SMCs but levels of depression were no longer significantly associated with SMCs. Adjusted $R^{2}$ showed an explained variance of $22 \%$.

\section{Discussion}

Levels of fatigue and anxiety were significantly and uniquely associated with SMCs, while levels of depression, insomnia, and pain intensity were not. The strong inter-correlations between the independent variables underlines the importance of accounting for all known comorbidities when trying to identify the predictors of SMCs. Levels of depression were significantly associated with SMCs when entered separately into the analysis, but it did not remain significant when levels of fatigue and anxiety were entered into the equation. This suggests that depression is not uniquely associated with SMCs; it rather seems to be secondary to symptoms of fatigue and anxiety.

Subjective memory complaints might reflect worries about one's own memory performance, making it an expression of anxiety. Previous studies have reported that memory related anxiety, experiencing anxious arousal in response to forgetting, becoming anxious when asked to remember something and worrying about one's own memory function corresponds to SMCs (Ponds and Jolles, 1996; Jorm et al., 2001; Mol et al., 2008). It remains unclear whether SMCs originate from dispositional tendencies to worry or cause worry due to increased vigilance of one's own cognitive performance, as our data do not provide any information about the cause and effect. SMCs may be the final product of the ongoing competition for attention. Anxiety consumes attention, which could be the underlying cause of SMCs because preoccupation with excessive thoughts of worry could disturb the processing of other information.

Our findings are in line with other studies that have reported a strong association between fatigue and SMCs (O'Boyle et al., 1990; Cope et al., 1995; Derouesne et al., 1999;

TABLE 2 | Inter-correlations between the symptom measures.

\begin{tabular}{|c|c|c|c|c|c|}
\hline & 1 & 2 & 3 & 4 & 5 \\
\hline (1) Insomnia & - & - & - & - & - \\
\hline (2) Pain & $0.334^{* *}$ & - & - & - & - \\
\hline (3) Fatigue & $0.248 * *$ & 0.146 & - & - & - \\
\hline (4) Depression & $0.266^{* *}$ & 0.052 & $0.276^{* *}$ & - & - \\
\hline (5) Anxiety & $0.268^{* *}$ & 0.088 & $0.177^{*}$ & $0.569^{* *}$ & - \\
\hline
\end{tabular}

*Correlation is significant at the 0.05 level 2-tailed, **Correlation is significant at the 0.01 level 2-tailed.

TABLE 3 | A summary of the statistical results.

\begin{tabular}{|c|c|c|c|c|c|c|}
\hline Variables & $\begin{array}{l}\beta \text {-estimate (Adjusted } \\
\text { age and gender) }\end{array}$ & $95 \% \mathrm{Cl}$ & $P$-value & $\begin{array}{l}P \text {-estimate (Adjusted } \\
\text { sign variables) }\end{array}$ & $95 \% \mathrm{Cl}$ & $P$-value \\
\hline Age & 0.002 & $(-0.012-0.016)$ & 0.321 & 0.003 & $(-0.010-0.017)$ & - \\
\hline Men vs. Women & 0.058 & $(-0.247-0.364)$ & 0.473 & 0.089 & $(-0.022-0.399)$ & - \\
\hline Fatigue +1 on CFS & 0.150 & $(0.095-0.206)$ & $<0.001$ & 0.153 & $(0.081-0.190)$ & $<0.001$ \\
\hline Anxiety + 1 on HADS & 0.073 & $(0.043-0.104)$ & $<0.001$ & 0.062 & $(0.027-0.097)$ & 0.001 \\
\hline Depression + 1 on HADS & 0.054 & $(0.018-0.091)$ & 0.004 & -0.009 & $(-0.050-0.032)$ & 0.524 \\
\hline Pain + 1 on SF8 & 0.027 & $(-0.288-0.342)$ & 0.969 & - & - & - \\
\hline Insomnia + 1 on ISI & 0.022 & $(-0.001-0.046)$ & 0.067 & - & - & - \\
\hline
\end{tabular}

Adjusted R-squared $=0.226$.

Robust multiple regression using Huber's correction was chosen for fatigue and in the fully adjusted model.

Bold values indicate significant findings. 
Williams et al., 2011). Chronic fatigue is characterized by the sensation of persistent and debilitating tiredness and exhaustion. While acute fatigue decreases and disappears after periods of rest, moderate to long-term fatigue does not respond to the same restorative techniques. Although the specific mechanisms underlying the association between fatigue and SMCs are unknown, one can speculate on whether the lower activation, sensations of tiredness, and exhaustion might explain this link. Fatigue might reduce the ability to mobilize and utilize effort to help regulate cognitive performance, contributing to the perception of cognitive decline.

Biased attention toward health-related threat stimuli and illness-related impairments among patients suffering from chronic fatigue has been reported (Ray et al., 1995; Aggrawal et al., 2006; Hou et al., 2008). The attentional bias toward signals of potential threat indicates some degree of anxiety, which is supported by the high comorbidity of fatigue and generalized anxiety disorder (Fischler et al., 1997). While effort is known to compensate for some of the cognitive difficulties associated with anxiety (Eysenck et al., 2007), symptoms of fatigue make it inherently difficult to mobilize and use the same compensational technique. The inability to tap into and utilize auxiliary processing resources might be the characteristic feature that differentiates fatigue from anxiety with respect to subjective memory functioning.

Both the distribution of diagnoses and measures on the selfreport questionnaires show that subjects present multiple nonspecific health-related complaints. Fatigue and chronic pain were the most common diagnoses followed by depression and anxiety. None of the subjects in the sample were referred based on a diagnosis of insomnia or sleep disturbances, still the mean score of the sample on ISI are within subthreshold insomnia. The mean score on the EMQ-R was higher than what has been found in MSpatients (Royle and Lincoln, 2008). This is somewhat surprising given the fact that the latter suffers from a neurodegenerative disease known to affect cognitive functioning (Bobholz and Rao, 2003) and brain morphology (Papadopoulos et al., 2009).

It has been reported that nurses in high stress wards with high scores on the Cognitive Failure Questionnaire had greater difficulties coping with stress and reported more emotional distress than nurses with low scores on the questionnaire (Broadbent et al., 1982). One study found that self-reported cognitive decline precedes work-stress-related exhaustion (Ekstedt and Fagerberg, 2005). High scores on selfreported cognitive dysfunction might indicate vulnerability in terms of experiencing work-related stress (Folkman et al., 1986). This line of research seems to support our notion that subjects experiencing fatigue might find it difficult to access and make use of cognitive resources in order to cope with or adjust to various demands of everyday life, this might in turn explain their

\section{References}

Aamland, A., Malterud, K., and Werner, K. (2012). Phenomena associated with sick leave among primary care patients with medically unexplained physical symptoms: a systematic review. Scand. J. Prim. Health Care 30, 147-155. doi: $10.3109 / 02813432.2012 .704812$ perceptions of impaired memory functioning. If SMCs reflect a lack of accessibility to cognitive resources, it might also affect coping and induce or increase stress and thereby become a catalyst for sick leave.

Our sample reported multiple symptoms, the co-occurrence of symptoms adds to the total symptom burden and the added morbidity is associated with sick leave (Aamland et al., 2012). SMCs are part of the rather complex equation and should be addressed in clinical practice. Self-report questionnaires measuring perceived memory functioning is a quick and easy way to introduce and assess SMCs in clinical practice. For some patients who report significant problems with memory it might be relevant to do a neuropsychological examination in order to get objective measures and assess specific cognitive functions. Some patients might also benefit from cognitive training. Some studies have reported promising results when it comes to the effects of cognitive training (Klingberg, 2010), while others have not (Shipstead et al., 2012).

Future research should examine the relationship between SMCs stress and coping, as it might provide new insight into important mechanisms with respect to occupational functioning and sick leave.

\section{Limitations}

The current study population is a small and selected sample, which limits the generalizability of the results. The results should be interpreted with caution as 16 of the originally eligible subjects had missing data on the EMQ and was excluded from the study. We used one item from SF8 Health Questionnaire to assess the level of pain. Considering that pain is a multidimensional experience we should have included more information in order to tap into the multifactorial nature of pain. The explained variance of the model was $22 \%$, which means that $78 \%$ of the variance when it comes to SMCs is explained by other factors than the ones examined in this study.

\section{Conclusion}

Subjective memory complaints should be included as part of the complex symptomology among patients who report multiple symptoms, especially in cases with symptoms of fatigue and anxiety. This knowledge should be incorporated into clinical practice and questionnaires assessing perceived memory failures may be an easy and effective way to get patients to communicate about their perceptions and experiences when it comes to this aspect of cognitive functioning and occupational health.

Our results also emphasize the importance of accounting for all known comorbidities when examining SMCs in patients with multiple symptoms.

Aggrawal, V. R., McBeth, J., Zakrzewska, J. M., Lunt, M., and MacFarlane G. J. (2006). The epidemiology of chronic syndromes that are frequently unexplained: do they have common associated factors? Int. J. Epidemiol. 35, 468-476. doi: 10.1093/ije/dyi265

Antikainen, R., Hanninen, T., Honkalampi, K., Hintikka, J., KoivumaaHonkanen, H., Tanskanen, A., et al. (2001). Mood improvement reduces 
memory complaints in depressed patients. Eur. Arch. Psychiatry Clin. Neurosci. 251, 6-11. doi: 10.1007/s0040601

Bastien, C. H., Vallieres, A., and Morin, C. M. (2001). Validation of the insomnia severity index as an outcome measure for insomnia research. Sleep Med. 2, 297-307. doi: 10.1016/s1389-9457(00)00065-64

Bjelland, J., Dahl, A. A., Haug, T. T., and Neckelmann, D. (2001). The validity of the hospital anxiety and depression scale an updated literature review. J. Psychosom. Res. 52, 69-77.

Bobholz, J. A., and Rao, S. M. (2003). Cognitive dysfunction in multiple sclerosis: a review of recent developments. Curr. Opin. Neurol. 16, 283-288.

Broadbent, D. E., Cooper, P. F., Fitzgerald, P., and Parkes, K. R. (1982). The cognitive failure questionnaire (CFQ) and its correlates. Br. J. Clin. Psychol. 21 1-16. doi: 10.1111/j.2044-8260.1982.tb01421.x

Chalder, T., Berelowitz, G., Pawlikowska, T., Watts, L., Wessely, S., Wright, D., et al. (1993). Development of a fatigue scale. J. Psychosom. Res. 37, 147-153. doi: 10.1016/0022-3999(93)90081-P

Cockshell, S. J., and Mathias, J. L. (2014). Cognitive functioning in people with chronic fatigue syndrome: a comparison between subjective and objective measures. Neuropsychology 28, 394-405. doi: 10.1037/neu00 00025

Comijs, H. C., Deeg, D. J. H., Dik, M. G., Twisk, J. W. R., and Jonker, C. (2002). Memory complaints; the association with psycho-affective and health problems and the role of personality characteristics. A 6-year follow-up study. J. Affect. Disord. 72, 157-165. doi: 10.1016/S0165-0327(01)00453-0

Cooper, C., Bebbington, P., Lindesay, J., Meltzer, H., McManus, S., Jenkins, R., et al. (2011). The meaning of reporting forgetfulness: a cross-sectional study of adults in the English 2007 Adult psychiatric morbidity survey. Age Aging 40, 711-717. doi: 10.1093/ageing/afr121

Cope, H., Pernet, A., Kendall, B., and David, A. (1995). Cognitive functioning and magnetic resonance imaging in chronic fatigue. Br. J. Psychiatry $167,86-94$. doi 10.1192/bjp.167.1.86

Derouesne, C., Lacomblez, L., Thibault, S., and Leponcin, M. (1999). Memory complaints in young and elderly subjects. Int. J. Geriatr. Psychiatry 14, 291-301.

Ekstedt, M., and Fagerberg, I. (2005). Lived experiences of the time preceding burnout. J. Adv. Nurs. 49, 59-67. doi: 10.1111/j.1365-2648.2004.03264.x

Erk, S., Spottke, A., Meisen, A., Wagner, M., Walter, H., and Jessen, F. (2011). Evidence of neuronal compensation during episodic memory in subjective memory impairment. Arch. Gen. Psychiatry 68:845-852. doi: 10.1001/archgenpsychiatry.2011.80

Eysenck, M. W., Derakshan, N., Santos, R., and Calvo, M. G. (2007). Anxiety and cognitive performance: attentional control theory. Emotion 7, 336-353. doi: 10.1037/1528-3542.7.2.336

Fimland, M. S., Vasseljen, O., Gismervik, S., By Rise, M., Halsteinli, V., Jacobsen, H. B., et al. (2014). Occupational rehabilitation programs for musculoskeletal pain and common mental health disorders: study protocol of a randomized controlled trial. BMC Public Health 14:368. doi: 10.1186/1471-245814-368

Fischler, B., Cluydts, R., De Gucht, V., Kaufman, L., and De Meileir, K. (1997). Generalized anxiety disorder in chronic fatigue syndrome. Acta Psychiatr. Scand. 95, 405-413. doi: 10.1111/j.1600-0447.1997.tb09653.x

Folkman, S., Lazarus, R. S., Dunkel-Schetter, C., Delongis, A., and Gruen, R. J. (1986). Dynamics of a stressful encounter: cognitive appraisal, coping and encounter outcomes. J. Pers. Soc. Psychol. 50, 992-1003. doi: 10.1037/00223514.50.5.992

Friedberg, F. (2010). Chronic fatigue syndrome, fibromyalgia, and related illnesses: a clinical model of assessment and intervention. J. Clin. Psychol. 66, 641-665. doi: $10.1002 /$ jclp.20676

Haug, T. T., Mykletun, A., and Dahl, A. A. (2004). The association between anxiety, depression, and somatic symptoms in a large population: the HUNT-II study. Psychosom. Med. 66, 845-851. doi: 10.1097/01.psy.0000145823.85658.0c

Holmen, J., Langballe, E. M., Midthjell, K., Holmen, T. L., Fikseaunet, A., Saltvedt, J., et al. (2013). Gender differences in subjective memory impairment in a general population. The HUNT study, Norway. BMC Psychol. 1:19. doi: 10.1186/2050-7283-1-19

Hou, R., Moss-Morris, R., Bradley, B. P., Peveler, R., and Mogg, K. (2008). Attentional bias towards health-threat information in chronic fatigue syndrome. J. Psychosom. Res. 65, 47-50. doi: 10.1016/j.jpsychores.2008. 03.008
Jorm, A. F., Christensen, H., Korten, A. F., Jacomb, P. A., and Henderson, A. S. (2001). Memory complaints as precursor of memory impairment in older people: a longitudinal analysis over 7-8 years. Psychol. Med. 31, 441-449.

Katz, R., Heard, A. R., Mills, M., and Leavitt, F. (2004). The prevalence and clinical impact of reported cognitive difficulties (fibrofog) in patients with rheumatic disease with and without fibromyalgia. J. Clin. Rheumatol. 10, 53-58. doi: 10.1097/01.rhu.0000120895.20623.9f

Klingberg, T. (2010). Training and plasticity of working memory. Trends Cogn. Sci. (Regul. Ed.) 14, 317-324. doi: 10.1016/j.tics.2010.05.002

Kronholm, E., Sallinen, M., Suutama, T., Sulkava, R., Era, P., and Partonen, T. (2009). Self-reported sleep duration and cognitive functioning in the general population. J. Sleep Res. 18, 436-446. doi: 10.1111/j.1365-2869.2009.00765

Landmark, P., Romundstad, P., Dale, O., Borchgrevink, P. C., and Kaasa, S. (2012). Estimating the prevalence of chronic pain: validation of recall against longitudinal reporting (the HUNT pain study). Pain 153, 1368-1373. doi: 10.1016/j.pain.2012.02.004

Landrø, N. I., Fors, E. A., Våpenstad, L. L., Holthe, Ø., Stiles, T. C., and Borchgrevink, P. C. (2013). The extent of neurocognitive dysfunction in a multidisciplinary pain center population. Is there a relation between reported and tested neuropsychological functioning?. Pain 154, 972-977. doi: 10.1016/j.pain.2013.01.013

Loge, J. H., Ekeberg, O., and Kaasa, S. (1998). Fatigue in the Norwegian population: normative data and associations. J. Psychosom. Res. 45, 53-65. doi: 10.1016/S0022-3999(97)00291-2

Lumley, M. A., Cohen, J. L., Borszcz, G. S., Cano, A., Radcliffe, A. M., Porter, L. S., et al. (2011). Pain and Emotion: a biopsychosocial review of recent research. J. Clin. Psychol. 67, 942-968. doi: 10.1002/jclp.20816

Magali, S. M., Sforza, E., Barthelemy, J. C., and Thomas-Anterion, C. (2012). Does subjective sleep affect cognitive function in healthy elderly subjects. Sleep Med. 13, 1146-1152. doi: 10.1016/j.sleep.2012.06.021

McCracken, L. M., and Iverson, G. I. (2001). Predicting complaints of impaired cognitive functioning in patients with chronic pain. J. Pain Symptom. Manage. 21, 392-396. doi: 10.1016/S0885-3924(01)00267-6

Mol, M. E. M., Ruiter, R. A. C., Verhey, F. R. J., Dijkstra, J., and Jolles, J. (2008). A study into the determinants of perceived forgetfulness: implications for future interventions. Aging Ment. Health 12, 167-176. doi: $10.1080 / 13607860801972503$

Morin, C. M., Belleville, G., Belager, L., and Ivers, H. (2011). The insomnia severity index: psychometric indicators to detect insomnia cases and evaluate treatment response. Sleep 34, 601-608.

Moriss, R., Wearden, A., and Mullis, R. (1998). Exploring the validity of the chalder fatigue scale in chronic faigue syndrome. J. Psychosom. Res. 45, 411-417. doi: 10.1016/S0022-3999(98)00022-1

Mowla, A., Ashkani, H., Ghanizadeh, A., Dehbozorgi, G. R., Sabayan, B., and Chohedri, A. B. (2007). Do memory complaints represent impaired memory performance in patients with major depressive disorder. Depress. Anxiety 0, 1-5.

Munoz, M., and Esteve, R. (2005). Reports of memory functioning by patients with chronic pain. Clin. J. Pain 21, 287-291. doi: 10.1097/01.ajp.0000173993.53733.2e

O'Boyle, M., Amadeo, M., and Self, D. (1990). Cognitive complaints in elderly depressed and pseudodemented patients. Psychol. Aging 5, 467-468. doi: 10.1037/0882-7974.5.3.467

Olssøn, J., Mykletun, A., and Dahl, A. (2005). The hospital anxiety and depression rating scale: a cross sectional study of psychometrics and case finding abilities in general practice. BMC Psychiatry 5:46. doi: 10.1186/1471-244X-5-46

Papadopoulos, D., Dukes, S., Patel, R., Nicholas, R., Vora, A., and Reynolds, R. (2009). Substantial archaecortical atrophy and neuronal loss in multiple sclerosis. Brain Pathol. 19, 238-253. doi: 10.1111/j.1750-3639.2008. 00177

Ponds, W. H. M., and Jolles, J. (1996). The abridged Dutch metamemory in adulthood (mia) questionnaire: structure and effect of age, sex and education. Psychol. Aging 11, 324-332. doi: 10.1037/0882-7974.11.2.324

Ray, C., Jefferies, S., and Weir, W. R. (1995). Coping with chronic fatigue syndrome: illness responses and their relationship with fatigue, functional impairment and emotional status. Psychol. Med. 25, 937-945. doi: 10.1017/S00332917000 37429

Roth, R. S., Geisser, M. E., Theisen-Goodvisch, M., and Dixon, P. J. (2005). Cognitive complaints are associated with depression, fatigue, female sex and 
pain catastrophizing in patients with chronic pain. Arch. Phys. Med. Rehabil. 86, 1147-1154. doi: 10.1016/j.apmr.2004.10.041

Royle, J., and Lincoln, N. B. (2008). The everyday memory questionnairerevised. Development of a 13-item scale. Disabil. Rehabil. 30, 114-121. doi: 10.1080/09638280701223876

Schotte, C. K. W., Bossche, B. V. D., Doncker, D. D., Claes, S., and Cosyns, P. (2006). A biopsychosocial model as a guide for psychoeducation and treatment of depression. Depress. Anxiety 23, 312-324. doi: 10.1002/da. 20177

Shipstead, Z., Redick, T., and Engle, R. (2012). Is working memory training effective. Psychol. Bull. 4, 628-654. doi: 10.1037/ a0027473

Smith, G. R. (1992). The epidemiology and treatment of depression when it coexists with somatoform disorders, somatization, or pain. Gen. Hosp. Psychiatry 14, 265-272. doi: 10.1016/0163-8343(92)90097-T

Stewart, R. (2012). Subjective cognitive impairment. Curr. Opin. Psychiatry 25, 445-450. doi: 10.1097/YCO.0b013e3283586fd8

Stewart, R., Besset, A., Bebbington, P., Brugha, T., Lindesay, J., Jenkins, R., et al. (2006). Insomnia comorbidity and impact and hypnotic use by age group in a national survey population aged 16-74 years. Sleep 11, 1391-1397.

Stewart, R., Godin, O., Crivello, F., Maillard, P., Mazoyer, B., Tzourio, Z., et al. (2011). Longitudinal neuroimaging correlates of sunbjective memory impairment: 4 year prospective community study. Br. J. Psychiatry 198, 199205. doi: 10.1192/bjp.bp.110.078683

Suhr, J. A. (2003). Neuropsychological impairment in fibromyalgia. Relation to depression, fatigue, and pain. J. Psychosom. Res. 55, 321-329.
Sunderland, A., Harris, J. E., and Baddely, A. D. (1983). Do laboratory tests predict everyday memory? A neuropsychological study. J. Verbal Learning Verbal Behav. 22, 727-738. doi: 10.1093/brain/awu207

Tesio, V., Torta, D. M., Colonna, F., Leombruni, P., Ghiggia, A., Fusaro, E., et al. (2014). Are fibromyalgia patients cognitively impaired? Objective and subjective neuropsychological evidence. Arthritis Care Res. 67, 143-150. doi: $10.1002 /$ acr. 22403

Ware, J. E., Kosinski, M., Dewey, J. E., and Gandek, B. (2001). How to Score and Interpret Single Item Health Status Measures: A Manual for Users of ohe Sf-8 Health Survey. Lincoln RI: Metric Incorporated.

Williams, D. A., Clauw, D. J., and Glass, J. M. (2011). Perceived cognitive dysfunction in fibromyalgia syndrome. J. Musculoskelet Pain 19, 66-75. doi: $10.3109 / 10582452.2011 .558989$

Zigmond, A. S., and Snaith, R. P. (1983). The hospital anxiety and depression scale. Acta Psychiatr. Scand. 67, 361-370. doi: 10.1111/j.1600-0447.1983.tb09716.x

Conflict of Interest Statement: The authors declare that the research was conducted in the absence of any commercial or financial relationships that could be construed as a potential conflict of interest.

Copyright (c) 2015 Aasvik, Woodhouse, Jacobsen, Borchgrevink, Stiles and Landrø. This is an open-access article distributed under the terms of the Creative Commons Attribution License (CC BY). The use, distribution or reproduction in other forums is permitted, provided the original author(s) or licensor are credited and that the original publication in this journal is cited, in accordance with accepted academic practice. No use, distribution or reproduction is permitted which does not comply with these terms. 